\title{
An Exploratory Study of Bicycle Sharing in Malaysia
}

\author{
Herwina Rosnan ${ }^{1}$, Nuraisyah Chua Abdullah² \\ ${ }^{1}$ Arshad Ayub Graduate Business, \\ ${ }^{2}$ Faculty of Law, \\ Universiti Teknologi MARA, Malaysia \\ herwinaros@gmail.com, nuraisyahc@yahoo.com
}

\begin{abstract}
In many countries, bicycle sharing service has been integrated into the urban public transportation system. This is because bicycle sharing is seen as a good alternative for the environment. Bicycle sharing is particularly popular for a short trip in urban areas where traffic congestion and pollution from vehicles' emission is a concern. In Malaysia, bicycle sharing service is relatively new. The primary objective of the study is to investigate public perceptions of the use of bicycle sharing. Data were collected through interview and secondary sources. The findings indicate that there are barriers that hinder the willingness of Malaysians to use bicycle sharing. Implications of the study are discussed.
\end{abstract}

Keywords: bicycle sharing; public transportation; quality of life; Malaysia

eISSN 2514-7528 @ 2019. The Authors. Published for AMER ABRA cE-Bs by e-International Publishing House, Ltd., UK. This is an open-access article under the CC BY-NC-ND license (http://creativecommons.org/licenses/bync-nd/4.0/). Peer-review under responsibility of AMER (Association of Malaysian Environment-Behaviour Researchers), ABRA (Association of Behavioural Researchers on Asians) and $c E-B s$ (Centre for EnvironmentBehaviour Studies), Faculty of Architecture, Planning \& Surveying, Universiti Teknologi MARA, Malaysia.

https://doi.org/10.21834/jabs.v4i12.327 


\subsection{Introduction}

Globally, bicycle sharing has become popular as an alternative to motorised public transport. A bicycle sharing system is a network of bicycles, which is also known as cycle-hire, public bicycle system, bike-share and cycle-share. Currently, the concept of bicycle sharing system is available in more than 200 countries. In many instances, bicycle sharing is promoted for short distance destination that connects the users to other public transport. In other words, bicycle sharing is associated with other public transport networks it serves as a connection for users to other public transportation such as train service. According to Wine (2012), a bicycle sharing system is a "network of bicycles" and kiosks that the public which include tourists and students can grab for a short distance trip and then return it to any designated station or dock in a different location. Harrah (2012) further explained that bicycle sharing system refers to bicycles which are not owned by individuals but service providers or operators that are placed in major cities, town, campuses or commercial areas that enable the community to access the service based on stipulated cost, time, distance of the trip as set by the operators or bicycle sharing service provider.

Although a bicycle as a mode of public transportation has long been practised in many countries, the concept of bicycle sharing is relatively new. According to DeMaio and Meddin (2012), in many urban areas, as a commercial transport system, a bicycle sharing system is indeed a relatively new practice. Renting a bicycle through bicycle sharing concept is a challenge to bicycle sharing service operators as many instances showed that without proper management, bicycle sharing is highly subject to public vandalism. As a result of technological advancement, many bicycle sharing operators have come up with various technologies to protect the businesses and increase the safety of the passengers. This includes using Global Position System (GPS) and Radio Frequency Identification (RFID) to track down the bicycles. Automation is now a backbone of the business with many features to increase the ease of use.

In 1998, France was the first country which introduced the first automated system by incorporating information and technology (ICT). The use of smart card makes it convenient for a user to collect the bicycle and easy drop-off at the station. Now, bicycle sharing is also referred to as 'Smart Bikes", "Bicycle Transit" and Public-Use Bicycle.

The concept of a bicycle sharing system involves rental scheme of bicycles where the user can collect the bicycle at any self-service station and leave it at any other bicycle dock designated by the service provided. This concept is similar to many car rental services except that bicycle sharing is meant for a short distance trip. The attraction of bicycle sharing concept is that users do not have to own a bicycle to commute and bicycle sharing provides a relatively cheaper and affordable mode of public transport.

Since it was first introduced, bicycle sharing has moved from merely a new exciting concept to some mainstream public transport alternatives. It receives growing attention in urban transportation (Vogel, Greiser, and Mattfeld, 2011). It has been widely used in major cities like London and Paris. It is estimated that there are 375 different bicycle sharing scheme all over the world and regionally there is around 236,000 usage of bicycle sharing with the largest consumption is in Hangzhou, China where 40,000 bicycle sharing usage was recorded (Wine, 2012). 


\subsection{The Evolution of Bicycle Sharing}

Since the introduction of bicycle sharing in the 1960s in Europe, there was a dramatic change in its service offerings. Bicycle sharing started in Amsterdam in 1965 followed by La Rochelle in 1976, and later bicycle sharing was introduced in Cambridge in 1993. These are the location where the concept of bicycle sharing in which user can borrow and return the bicycle at any location was introduced. However, in the early operations, there were no measures taken to ensure the bikes are used and cared of. Cases of vandalism and theft make the service offerings unattractive to the operators. In 1991, the second generation of bicycle sharing was introduced in Denmark that able to overcome the issue of theft and vandalism (DeMaio, 2009). Then the third generation was later introduced in 1995, where the bicycle sharing services were offered in Copenhagen on a large scale. By 1995, the first large scheme was launched in Copenhagen. The new project emulated "lending library" scheme with an annual fee or membership fee charge to the users (Metrolinx, 2009). To reduce theft, the service provider or operators built a non-standard component. The bicycle was also a heavy duty bicycle compared to a normal bicycle to withstand frequent usage. The new technology built was simple and cheaper to install. Some operators used manual tracking but most used coin-operated locks to lock the bicycle. Although the system was better than the previous system, it was only able to reduce theft and vandalism. Hence, in the 1990s, a more reliable system of smartcard was introduced with a more reliable feature to protect the interest of the operators and the safety of the passengers. The first use of smart card technology in bicycle sharing schemes was introduced in 1998. These third generation of bicycle sharing are more sophisticated in that it is not only improved in the bicycle features but also the use of automatic features for payment systems and bicycle locking. Global Positioning System (GPS) and Radio Frequency Identification (RFID) technologies were among popular system that enables operators to track down the bicycles which ultimately control vandalism and theft. Websites are also important tools to provide real-time information on the availability of the bicycle at any stations. In most instances, bicycle sharing service is a collaborative effort of private and public partnership.

\subsection{Bicycle Sharing in Malaysia}

Bicycle sharing system was introduced in Penang as an alternative mode of getting around Georgetown (a heritage city) without driving. The service intends to help in reducing the number of cars on the road within the popular tourists' attraction. Further, the development of the Malaysian economy is an opportunity for the establishment of a bicycle sharing service business. With the advancement of public transportation systems in Malaysia, makes the concept of bicycle sharing relevant to be introduced to help the users to access the public transit network. Liu, Jia and Cheng (2012) termed the new solution as "the last mile solution". The concept of bicycle sharing can now be found in a major city of Kuala Lumpur, Selangor, Johor and Terengganu. In 2017, more foreign bicycle sharing system operators entered the Malaysian market.

However, bicycle sharing is a new concept in Malaysia, and unlike other countries, the readiness and acceptance of bicycle sharing concept have yet to be known. Companies like O'Bike found it challenging to serve the new market as the understanding, culture and 
awareness of the public are not identified. Cycling culture in Malaysia is usually for leisure activity purposes and sports and not as a mode of transportation. Thus, the primary objective of this study is to seek an understanding of the use of bicycle sharing as part of the public transportation system. This study is an exploratory study where data is gathered mainly to provide an in-depth understanding of the public perception of the bicycle sharing system in Malaysia.

\subsection{Literature Review}

Bicycle sharing has received academic attention since the new scheme was first introduced. However, there were conflicting findings on the success of bicycle sharing on users, business operators and the cities in general. For example, Study by Liu et al. (2012) in Beijing city found declining popularity of bicycle sharing, while a study by Roman and Roman (2014) in Warsaw city found an increasing prevalence and increasing number of users. However, the fact the bicycle sharing system posed a positive characteristic particularly in promoting sustainable mobility, the exploitation of bicycle sharing business remain attractive (Bordagaray, Ibeas and Olio, 2012).

In a country where bicycle sharing concept is new, studies were focused on the perception of the public or users on bicycle sharing services (for example, Bordayaray et al., 2012 and De Sousa et al., 2014). The concept of bicycle sharing is deemed beneficial to all in that it is good for the environment (non-pollutant), good for the users (healthy lifestyle) and good for the cities (less congestion on motorised traffic). However, there are indeed many barriers to the usage of the bicycle. De Sousa et al. (2014) found that the infrastructure plays an essential factor for bicycle sharing usage. The inadequacy of dedicated bicycle infrastructure posed a significant barrier that impedes the utilisation of bicycle sharing in Brazil. The study also found that the perceived barrier differs between cities in which slopes become the second critical barrier in Sao Paulo city while security and climate was the second crucial barrier for Campo Grande city. Although infrastructure was found as an essential factor that discourages bicycle sharing, it was beyond the capability of business operators to handle it as the local government plays an important role to ensure proper infrastructures and public facilities. This factor is the reason why most countries enter into private-public partnership when it comes to bicycle sharing service offerings. For example, in Germany, Netbike and Norisbike are working together providing self-service bike central to support urban transport as well as to promote urban tourism where the German Ministry of Transport endorsed the project.

Previous studies found barriers that hinder the usage of bicycle sharing includes lack of infrastructure like dedicated bicycle track, safety, weather, geographical landscape, the distance of the trip, physical fitness and inconvenience (Fishman, 2012; De Sousa et al., 2014). Studies also conducted to identify gender difference in cycling where males were found to be a more frequent to cycle that female. The reason is related to factors like convenience where women are associated with activities like shopping, transporting kid to the school where the usage of the private car is a necessity. Furthermore, women are also 
associated with higher conscious towards safety and are more particular about the appearance to work (Garrard et al., 2008). Nevertheless, in the country with a high level of cycling, there is a balanced number of men and women bicycle cyclists like in the Netherlands (Garrard, 2003). Concerning age, Parkin (2004) found that a higher proportion of people who are under 34 years old cycle more. This phenomenon could be related to the physical nature of the younger age, lower level of car ownership factor and the tendency of younger people to live in the cities area.

In a new location where bicycle sharing is a new initiative, scholars conducted studies to investigate the perceptions of the public towards bicycle sharing. In this context, Davis's model (1989) of technology adoption (TAM) carries two theoretical constructs that are relevant in examining the perception of users on bicycle usage. These two constructs are perceived usefulness and perceived ease of use. Both constructs are seen to impact the intention to use technology directly. Perceived usefulness is related to the people's perception of how technology facilitates them in carrying out their task. The authors indicate that the intention of users to use system can be measured using these two constructs of perceived ease of use and perceived usefulness as it is suggested that both are predominantly necessary measures (Davis, 1989; Lee, 2010; Vankatesh and Davis, 1996). Hence, TAM is a model that emphasises on perceived ease of use and perceived usefulness as two fundamental determinants of technology acceptance by users (Davis, 1989). The definition of Perceived Ease of Use as defined by Davis (1989) is "the degree to which a person believes that using a particular system would be free of effort" and hypothesized that perceived ease of use has a positive effect on perceived usefulness as "the degree to which a person believes that using a particular system would enhance his or her job performance".

\subsection{Perceived Usefulness}

It has been found in a study of bicycle sharing system, that this project can lead to reduced demand for parking space, reduced effect to greenhouse emission and reduced traffic congestion, thereby helping minimise resource consumption and nurture a greener environment in campus (Ashley, 2012). Furthermore, cycling is associated with a healthy lifestyle and increases the health of a person. It is also linked to the barriers of bicycle sharing usage with the common concerns about the climate, travelling distance, traffic congestion, crowding of cycling path, the use of GPS while cycling and the automatic payment system. According to the TAM model and other related literature, attitude and a behavioural intention can be fostered by perceived usefulness (Lee, 2015). When people feel that the commercial bicycle sharing systems are useful and beneficial to them, they will develop a positive view toward the bike-sharing system, and be more willing to use the system. Since bicycle sharing is using a special application to operate their bicycle, it can relate to the literature that a mobile application (mobile apps) is generally perceived as more useful provided the usage is easy (Wenga et al., 2017). In the other study by Hussain and Mkpojiogu (2016), they describe perceived of usefulness in a context where the user may accept that a given innovation such as a mobile map is helpful, but while using the mobile, the user may find out that the innovation may be difficult to use. For instance, the object on the mobile screen may be difficult to see. 


\subsection{Perceived Ease of Use}

Users' intention of technology adoption can be measured by perceived ease of use of the technology (Davis, 1989). This denotes that individuals believe the use of services or products is effortless, that using particular product/service would be free from effort. For example, utilising a specific technology like the mobile map would be free of physical and mental exertion. In the same token, applying the same concept in the context of application technology, perceived ease of use is inferred to as the degree to which users perceive the ease of interface with application allows them to obtain the appropriate and useful information they required (Rahman et al., 2013). This statement is consistent with Yuslihari et al. (2011) findings which suggested that perceived ease of use has a positive and significant influence towards consumers' attitude on online purchasing. Islam and Salma (2016) stated that there is a positive causality between perceived ease of use and usage intention. Ease of use is the user's impression of the measure of requirement needed to use a technology or the level to which user accepts that applying a specific innovation will be easy and effortless (Chen, 2016). Apart from Davis et al. (1989), Chen (2016) also included perceived ease of use as a precursor to intention to use.

\subsection{Methodology}

This study is an exploratory study where data collection was conducted through the interview. The primary objective of this study is to gain an in-depth understanding of the public perceptions of bicycle sharing system. Hence, the focus is to seek opinion and description rather than theory formation. Bicycle sharing businesses in Malaysia are mostly located at train stations. Therefore, respondents from this location were approached. Among the respondents, few have experiences in using bicycle sharing service while the majority of them have no experience at all. A total of 18 respondents were willing to participate in the study.

The interview sessions were recorded, and data were transcribed. The study employed a semi-structured interview. Most of the interview sessions lasted less than half an hour as most of the respondents were public transport commuters who were waiting to ride public transport. The interview transcripts were vigorously read, and notes were created to identify emerging patterns. The analysis was done manually by comparing the transcripts to look at similarities and differences of information. The analysis was not done verbatim as the aim of the study is to look for significant ideas related to the phenomenon under study. Content analysis was also conducted to analyse secondary data. Statistical validity is not typical of qualitative research rather; an elaborate and rich data offers valuable information that provides an in-depth understanding of the phenomenon.

\subsection{Results}

The study seeks to investigate public perceptions of the usage of bicycle sharing service integrated into the public transportation system in Malaysia. The analysis of data from the interviews conducted resulted in some consistent patterns. Significant opinions on bicycle sharing cited barriers to using bicycle sharing as a public mode of transportation. The initial 
question on the familiarity of the respondents towards the concept of bicycle sharing system found that most respondents are aware of the offerings of bicycle sharing service, but they do not understand the concept of bicycle sharing introduced by business operators. Some respondents who have had experience in bicycle sharing lamented over the lack of bicycle infrastructure where limited bicycle path has been a major concern. Besides, bicycle and pedestrian are commonly sharing the same road which leads to safety issues to both rider and pedestrian.

According to Chen (2016) perceived ease of use is a precursor to the intention to use. With regards to intention to use bicycle sharing, most respondents indicate the unwillingness to embark on bicycle sharing service. The aversion of the public to use bicycle sharing service is attributed to several factors which include the weather. Malaysia's hot and humid weather makes it inconvenient for the general public to ride a bicycle mainly to work. On top of that, the use of a bicycle is not convenient during raining season. Few female respondents questioned the appropriateness for them to ride a bicycle if they are wearing formal office attire particularly the baju kurung which is an official national attire in Malaysia. Consistent with previous findings by Garrard (2003), appearance at work is another factor that hinders female from using a bicycle to work. Compared to other public transport, respondents found that the use of a bicycle is less convenient based on the factors mentioned above. According to the technology acceptance model (TAM), attitude and a behavioural intention can be fostered by perceiving usefulness (Lee, 2015). In other words, if people feel that bicycle sharing is useful and beneficial to them, they will develop a positive view and more willing to use them. The current study indicates otherwise, where respondents highlighted barriers to use bicycle sharing service.

Although it was not explicitly mentioned during the interview, the respondents indicated that bicycle sharing culture is still uncommon to citizens. Indeed, in Malaysia, although there is a positive trend in bicycle usage, it is mostly for leisure and sports.

One respondent said that since she was raised in the city, there were limited space to ride a bicycle and that was the reason she cannot ride a bicycle. Although this does not represent the views of others, the future study may want to look at the profile of the respondents in the urban area and verify whether the inability to ride a bicycle hinders the effort towards promoting bicycle sharing service.

\subsection{Discussion}

The finding shows that most of the respondents do not appreciate the initiative towards the usage of bicycle sharing service as they do not think this is a feasible idea. Moreover, lack of bicycle path makes it inconvenient for the public to use the bicycle sharing service as one of their main transportation modes. This finding is consistent with previous studies by Fishman (2012) and De Sousa et al. (2014). It is difficult for the cyclist to find the correct road to cycle on because bicycle stands at a weird middle ground between transportation and pedestrians. On top of that, the local climate is one of the main factors the public is hesitant to use the bicycle as a mode of transportation.

Bicycle sharing system is uncommon to most Malaysian as it is not a culture to use a 
bicycle as a medium of commuting from places to places. Cycling culture in Malaysia is usually for leisure activities purposes and sports. Besides, hot weather in Malaysia makes the public to think twice on whether they should use a bicycle as an alternative mode of transportation. The general public does not understand the rationale behind the introduction of bicycle sharing initiatives in Malaysia as they do not think that this is an excellent idea for them to use it.

Local newspaper, the Star Online (June, 2017) reported that there is an increasing number of bicycle sharing users in Malaysia as indicated in the growing number the bicycle sharing applications adopted. Currently, it is estimated that there are 20,000 users. The rental is based on usage in which every 15 minutes usage the user will be charge RM1. However, the number of users decreasing by 10 per cent every quarter of the month since public usually want to give it a try as a first timer and not repeating for the next time. They would instead take Grab, Uber or taxi to their destination rather than using a bicycle sharing service. The issue of a decreasing number of users is not unique to Malaysia's market which is relatively new in bicycle sharing. In a more matured market like Beijing, they are also experiencing a decreasing number of bicycle sharing user as these users prefer other modes of public transport.

Moreover, due to lack of education on the bicycle sharing system in Malaysia, users do not become aware of its parking system which causes them to park everywhere and disrupt the traffic. According to a local mainstream newspaper, the New Straits Times (September 2017) reported that Petaling Jaya City Council (MPAJ) enforcement officers seized more than 250 bicycles found on the sidewalks and streets of Petaling Jaya (MBPJ). The bicycles were seized because there were obstructions on the road. Further to seizure, the bicycle sharing service provider had been fined RM17,000 by Petaling Jaya City Council (MBPJ). The bicycle sharing has been instructed by the MBPJ to remove all its existing bicycle in Selangor except in Subang Jaya as Subang Jaya will introduce a pilot project for bicycle sharing system beginning October 2017. MPPJ also claimed that the dockless idea makes the bicycle too messy.

Bicycle theft and vandalism are common in other countries, and Malaysia is not an exception. Malaysian Digest (November, 2017) reported that bicycles were found in inappropriate places such as throwing in drains and bushes, left in hidden places in housing areas and even hanging from signboards. Vandalism of the bicycles can be found in missing paddles, handles and even tires. This affects the offerings of bicycle sharing service where the service providers have to come up with more stringent terms of bicycle sharing.

On the impact of the bicycle sharing scheme to the cities, Buhrmann (2008) stated that there is little impact which has been reported. The only noticeable effect would be the increasing number of bicycle sharing schemes. The author also said that in Barcelona, Leon and Paris, bicycle sharing posed a little impact on the effort to reduce car on the road. It was noted that the percentage of a motorised trip that is replaced by bicycle sharing is merely an average of 2-4 per cent. However, Shaheen et al. (2010) reported that the user of bicycle increased by 44 per cent in Lyon when it was first introduced. Meanwhile, Paris is experiencing a 70 per cent increase of user after bicycle sharing was launched. Although there is a limited data the impact of bicycle sharing, a survey on user in 2008 revealed that 
90 per cent of the respondents said that "bicycle sharing allowed them to make trips that would he otherwise been impossible" while 20 per cent of respondents agree that with bicycle sharing reduced car usage (Shaheen et al., 2010).

Bicycle sharing service providers are still facing vandalism issue. Shaheen et al. (2010) further reported that in European Counties, Paris is experiencing the most number of vandalism cases despite the use of sophisticated technology to reduce the problem. According to the report "within the first two years of operations, nearly the whole system fleet of 20,600 bicycles had to be replaced at the cost of 400 Euros per bicycle. Some 7,800 bicycles were stolen, and 11,600 bicycles were vandalised beyond repair." The findings of this research also indicate vandalism as one of the main problems faced by service operators. The attitude of the users is also a challenge that requires the service operators to introduce more stringent terms and conditions of usage. However, too many rules imposed on the users may hamper the ease of use of the bicycle that eventually could draw the users away.

\subsection{Conclusion and Implication}

Bicycle sharing system is a modern public transport scheme that has the potential to be developed. Theoretically, it provides a sustainable public transport alternative and plays an integral part in increasing viable transport options in cities. The understanding of the behaviour and perceptions of the public towards bicycle sharing would be able to upgrade the bicycle sharing service provider and contributes to the creation of a sustainable city. Policy can be directed towards educating and promoting the growth of sustainable and environmentally friendly public transport system. In this context, the role of government is critical in ensuring the success of the bicycle sharing system. Based on a study by Liu et al. (2012) the authors found out that when the China government intervenes and encourage the users to commute by bicycle, the number of bicycles sharing users in China increased tremendously. This was when the government restricted the used of motorised vehicles to accommodate for the Olympic Games. However, after the event, there was a sharp drop in the number of bicycle commuters which also affect the business of the service providers. Some of the bicycle sharing service operators had to close down their business as users prefer other modes of public transport.

The benefit of bicycle sharing system should not be overrated. Although theoretically, it could reduce congestion, provide a healthy lifestyle for the users and offer an environmentally-friendly mode of transport, the cost of establishing the system need to be deliberate. The local administrative authority plays an essential role in supporting the initiative. This is because a successful model of bicycle sharing system takes a lot more than merely promoting its advantages. For example, in the Netherlands, the bicycle sharing system is integrated into a more comprehensive public transport that increases the efficiency of bicycle usage. This model integration (Britton, 2009) is a self-promoting tool to increase the utilisation of bicycle sharing. The understanding of the behaviour and perceptions of the public towards bicycle sharing would be able to upgrade the bicycle sharing industry and contributes towards the creation of the sustainable city. Policy can be directed towards 
educating and promoting public transportation, which is environmentally-friendly.

Since many bicycle sharing operators have penetrated many local universities in Malaysia, it is suggested that future research investigate the implementation of this initiative among university students. This is because the success of this program is instrumental and would be an indication of the growth of bicycle sharing sector in Malaysia.

\section{Acknowledgement}

The authors would gratefully acknowledge the Institute of Research Management and Innovation (IRMI) Universiti Teknologi MARA (UiTM), Shah Alam and Ministry of Higher Education Malaysia (MOHE) for the financial support through the Fundamental Research Grant Scheme, File No: 600-IRMI/FRGS 55/3(36/2015).

\section{References}

Ashley, J. (2012). Bike sharing as alternative transportation at Bridgewater State University. Undergraduate Review, 8(1), 16-25.

Bordagaray, M., Ibeas, A., \& dell'Olio, L. (2012). Modelling user perception of public bicycle services. ProcediaSocial and Behavioral Sciences, 54, 1308-1316.

Britton E. 2009. Public Bikes in Latin American Cities: great idea but what next?

Bührmann, S. (2008). Bicycles as public-individual transport-European developments. Köln, Rupprecht Consult Forschung und Beratung $\mathrm{GmbH}$.

Chan, Dawn (2017, September 13). MBPJ slaps oBike with RM17,000 fine for obstruction of public spaces. News Straits Times. Retrieved from https://www.nst.com.my/news/nation/2017/09/279335/mbpj-slaps-obike-rm17000fine-obstruction-public-spaces.

Chen, S. Y. (2016). Green helpfulness or fun? Influences of green perceived value on the green loyalty of users and non-users of public bikes. Transport Policy, 47, 149-159.

Davis, F. D. (1985). A technology acceptance model for empirically testing new end-user information systems: Theory and results (Doctoral dissertation, Massachusetts Institute of Technology).

Davis, F. D., Bagozzi, R. P., \& Warshaw, P. R. (1989). User acceptance of computer technology: a comparison of two theoretical models. Management Science, 35(8), 982-1003.

DeMaio, P. (2009). Bike-sharing: History, impacts, models of provision, and future. Journal of public transportation, 12(4), 3.

DeMaio P and Meddin R (2012) The Bike-sharing World Map.

DeMaio, P. (2009). Bike-sharing: History, impacts, models of provision, and future. Journal of public transportation, 12(4), 3.

de De Sousa, A. A., Sanches, S. P., \& Ferreira, M. A. (2014). Perception of barriers for the use of Bicycles. ProcediaSocial and Behavioral Sciences, 160, 304-313. 
Fishman, E. (2011). Evaluating the benefits of public bicycle schemes needs to be undertaken carefully. British Medical Journal, 343(d4521).

Franco, L. P. C., Campos, V. B. G., \& Monteiro, F. B. (2014). A characterisation of commuter bicycle trips. ProcediaSocial and Behavioral Sciences, 111, 1165-1174.

Garrard, J. (2003). Healthy revolutions: promoting cycling among women. Health Promotion Journal of Australia, 14(3), 213-215.

Garrard, J., Rose, G., \& Lo, S. K. (2008). Promoting transportation cycling for women: the role of bicycle infrastructure. Preventive medicine, 46(1), 55-59.

Harrah, K. (2012). Utilising bicycles to promote physical activity. VAHPERD Journal, 33(2), 4-6.

Horton, D., Rosen, P., \& Cox, P. (Eds.). (2016). Cycling and society. Routledge.

Hussain, A., Mkpojiogu, E. O., \& Yusof, M. M. (2016, August). Perceived usefulness, perceived ease of use, and perceived enjoyment as drivers for the user acceptance of interactive mobile maps. In AIP Conference Proceedings (Vol. 1761, No. 1, p. 020051). AlP Publishing.

Islam, K. A., \& Salma, U. (2016). Mobile Banking Operations and Banking Facilities to Rural People in Bangladesh. International Journal of Finance and Banking Research, 2(4), 147.

Khor, Samantha (2017, November 21). Irresponsible oBike Users Are Vandalising And Dumping Bicycles Across Klang Valley. Retrieved from https://says.com/my/news/obike-bicycles-found-damaged-parts-stolen-dumped-inwater-like-rubbish-klang-valley.

Lee, J.W. (2010). The roles of demographics on the perceptions of electronic commerce adoption. Academy of Marketing Studies Journal, 14(1), 71-89.

Liu, Z., Jia, X., \& Cheng, W. (2012). Solving the last mile problem: Ensure the success of public bicycle system in Beijing. Procedia-Social and Behavioral Sciences, 43, 73-78.

Meddin, R. (2011). The bike-sharing world: First days of summer 2011. 2011 [2012-12-12]. http://bike-sharing. blogspot com/search.

Meddin, R., \& DeMaio, P. (2014). The bike-sharing world: First week of June 2011. 2011 [2012-12-12]. http://bikesharing. blogspot. com.

METROLINX (2009). Bike Share Program Investigation - Best Practices Investigation. Toronto.

Midgley, P. (2011). Bicycle-sharing schemes: enhancing sustainable mobility in urban areas. United Nations, Department of Economic and Social Affairs, 1-12.

Nair, Vijenthi (2017, June 15). Bike-sharing service already has 20,000 users. Star Online, Retrieved from https://www.thestar.com.my/metro/community/2017/06/15/bikesharing-service-already-has-20000-users/.

Parkin, J. (2004) Determination and Measurement of Factors Which Influence Propensity to Cycle to Work. PhD Thesis, Institute for Transport Studies, University of Leeds.

Roman, M., \& Roman, M. (2014). Bicycle transport as an opportunity to develop urban tourism-Warsaw example. Procedia-Social and Behavioral Sciences, 151, 295-301. 
Rosnan, H., \& Abdullah, N.C. / Journal of ASIAN Behavioural Studies (jABs), 4(12) Jan / Apr 2019 (p. 25-36)

Shaheen, S., Guzman, S., \& Zhang, H. (2010). Bikesharing in Europe, the Americas, and Asia: past, present, and future. Transportation Research Record: Journal of the Transportation Research Board, (2143), 159-167.

Venkatesh, V., \& Davis, F. D. (1996). A model of the antecedents of perceived ease of use: Development and test. Decision Sciences, 27(3), 451-481.

Vogel, P., Greiser, T., \& Mattfeld, D. C. (2011). Understanding bike-sharing systems using data mining: Exploring activity patterns. Procedia-Social and Behavioral Sciences, 20, 514-523.

Wine, J., \& Easy, B. (2012). Bicycle Share Feasibility Study New Orleans. 